\title{
Relative Manin-Mumford for Abelian Varieties
}

D. W. Masser

Institute of Mathematics

Preprint No. 2014-15

University of Basel

November 2014

CH - 4051 Basel

Switzerland

$\underline{\text { www.math.unibas.ch }}$ 


\title{
Relative Manin-Mumford for abelian varieties
}

D. Masser

2010 MSC codes. 11G10, 14K15, 14K20, 11G50, 34M99.

\begin{abstract}
With an eye or two towards applications to Pell's equation and to Davenport's work on integration of algebraic functions, Umberto Zannier and I have recently characterised torsion points on a fixed algebraic curve in a fixed abelian scheme of dimension bigger than one (when all is defined over the algebraic numbers): there are at most finitely many points provided the natural obstacles are absent. I sketch the proof as well as the applications.
\end{abstract}

A very simple problem of Manin-Mumford type is: find all roots of unity $\lambda, \mu$ with $\lambda+\mu=1$. Here the solution is easy: we have $|\lambda|=|1-\lambda|=1$ and so in the complex plane $\lambda$ lies on the intersection of two circles; in fact $\lambda$ must be one of the two primitive sixth roots of unity (the picture doesn't work too well in positive characteristic, and indeed any non-zero element of any finite field is already a root of unity, so from now on we stick to zero characteristic). This result has something to do with the multiplicative group $\mathbf{G}_{\mathrm{m}}$, which can be regarded as $\mathbf{C}^{*}$. Actually with $\mathbf{G}_{\mathrm{m}}^{2}$ and the "line" inside it parametrized by $P=(\lambda, 1-\lambda)$ : we ask just that $P$ is torsion.

Now it is easy to generalize, at least the problem, to other algebraic varieties in other commutative algebraic groups.

For example let $E$ be the elliptic curve defined by $y^{2}=x(x-1)(x-4)$. Asking for all complex $\lambda$ such that the points

$$
(2 \lambda, \sqrt{2 \lambda(2 \lambda-1)(2 \lambda-4)}), \quad(3 \lambda, \sqrt{3 \lambda(3 \lambda-1)(3 \lambda-4)})
$$

are both torsion amounts to asking for torsion points on a certain curve in the surface $E^{2}$. But here the solution is much more difficult (and it is not clear to me that one can find all $\lambda$ explicitly as above). 
It was Hindry $[\mathrm{H}]$ who solved the general problem with any algebraic variety in any commutative algebraic group $G$. The outcome for a curve in $G$ is that it contains at most finitely many torsion points unless one of its components is a connected one-dimensional "torsion coset"; that is, a translate $P_{0}+H$ of an algebraic subgroup $H$ of $G$ by a torsion point $P_{0}$. This $H$ contains infinitely many torsion points and so $P_{0}+H$ also.

Thus for $G=\mathrm{G}_{\mathrm{m}}^{2}$ the analogue for $\lambda \mu=1$ of the problem above will not lead to finiteness, as the curve is such an $H$. Similarly $\lambda \mu=-1$ is $P_{0}+H$ for $P_{0}=(1,-1)$ with $2 P_{0}=0$ (written additively).

More generally $G$ can be $\mathbf{G}_{\mathrm{m}}^{n}, E^{n}$ as in Habegger's talks in this volume, or an abelian variety $A$ as in Orr's article, or products of these, or "twisted products" sitting inside an exact sequence

$$
0 \rightarrow T \rightarrow G \rightarrow A \rightarrow 0
$$

where $T$ is a power of $\mathbf{G}_{\mathrm{m}}$ or even a product $\mathbf{G}_{\mathrm{a}}^{r} \times \mathbf{G}_{\mathrm{m}}^{s}$ for the additive group $\mathbf{G}_{\mathrm{a}}=\mathbf{C}$. Here the twisting can be quite complicated and $G$ can end up very far from just $T \times A$. It is classical that every commutative algebraic group over $\mathbf{C}$ has this form. We will see how several types turn up in applications.

The applications involve most naturally the "relative case", where $G$ itself is allowed to vary in a family. Most of the current results allow only a single parameter here, and we already have parameters in the algebraic variety, so this had better stay a curve, essentially with a parameter $\lambda$, and $G$ had better depend on no more than $\lambda$. An example like (1) involves the points

$$
(2, \sqrt{4-2 \lambda}), \quad(3, \sqrt{18-6 \lambda})
$$

now on the elliptic curve $E_{\lambda}$ defined by $y^{2}=x(x-1)(x-\lambda)$; that is the famous Legendre family. Again we go to $E_{\lambda}^{2}$, where the square is the "fibre square" defined by the equations

$$
y_{1}^{2}=x_{1}\left(x_{1}-1\right)\left(x_{1}-\lambda\right), \quad y_{2}^{2}=x_{2}\left(x_{2}-1\right)\left(x_{2}-\lambda\right)
$$

with $\lambda$ in common, and we get a curve defined by $x_{1}=2, x_{2}=3$. Then by [MZ1] there are at most finitely complex values of $\lambda$ (now not 0,1 so that we have a genuine elliptic curve) such that both points in (3) are torsion. Here their effective determination may be a difficult problem in practice and even in principle.

In various works Umberto Zannier and I have treated any curve in any parametrized abelian variety $A_{\lambda}$ of "relative dimension" at least two, sometimes with the proviso that everything is defined over the field $\overline{\mathbf{Q}}$ of all algebraic numbers. We get finiteness with 
a similar condition about torsion cosets, now interpreted schemewise or more intuitively "identically in $\lambda$ ". For example in $E_{\lambda}^{2}$ above these are defined essentially by the vanishing of non-trivial integral linear combinations $n_{1}\left(x_{1}, y_{1}\right)+n_{2}\left(x_{2}, y_{2}\right)$. It is not difficult to show that this is impossible for (3). On the other hand

$$
2(0,0)=2(1,0)=2(\lambda, 0)=4(\sqrt{\lambda}, i(\lambda-\sqrt{\lambda}))=0
$$

so it is not quite easy.

A start has been made on more general $G_{\lambda}$. First with Bertrand and Pillay we have considered

$$
1 \rightarrow \mathbf{G}_{\mathrm{m}} \rightarrow G_{\lambda} \rightarrow E_{\lambda} \rightarrow 0
$$

and

$$
1 \rightarrow \mathbf{G}_{\mathrm{m}} \rightarrow G_{\lambda} \rightarrow E \rightarrow 0
$$

with $E$ not depending on $\lambda$. Bertrand [Be] had already given a surprising counterexample in (5): in rather special situations it is possible to construct what he calls a "Ribet curve" having infinitely many torsion points, even though it is not a torsion coset. We then checked in [BMPZ] that this happens only for Ribet curves.

And Harry Schmidt in Basle [Sc] has done

$$
0 \rightarrow \mathbf{G}_{\mathrm{a}} \rightarrow G_{\lambda} \rightarrow E_{\lambda} \rightarrow 0
$$

where it is reassuring to find that there are no counterexamples.

We give a short proof sketch of the result for (3). As in Habegger's talks, it hinges on the analytic representation of an elliptic curve as a quotient of $\mathbf{C}$ by a lattice, as in the general strategy of Zannier expounded in [PZ]. For $E_{\lambda}$ this lattice $\Omega_{\lambda}$ depends on $\lambda$, and in fact one can take a basis of periods

$$
\Omega_{\lambda}=\mathbf{Z} f_{\lambda}+\mathbf{Z} g_{\lambda}
$$

where $f_{\lambda}$ is hypergeometric $\pi F\left(\frac{1}{2}, \frac{1}{2}, 1 ; \lambda\right)$ and $g_{\lambda}=i f_{1-\lambda}$. The points $(3)$ correspond in $\mathbf{C}$ to "elliptic logarithms" $u_{\lambda}, v_{\lambda}$, say; and since $\mathbf{C}=\mathbf{R} f_{\lambda}+\mathbf{R} g_{\lambda}$ there are real functions $p_{\lambda}, q_{\lambda}, r_{\lambda}, s_{\lambda}$ with

$$
u_{\lambda}=p_{\lambda} f_{\lambda}+q_{\lambda} g_{\lambda}, \quad v_{\lambda}=r_{\lambda} f_{\lambda}+s_{\lambda} g_{\lambda}
$$

As $\lambda$ moves, the locus of $z_{\lambda}=\left(p_{\lambda}, q_{\lambda}, r_{\lambda}, s_{\lambda}\right)$ has real dimension two in $\mathbf{R}^{4}$ and is in fact a (sub-)analytic surface $Z$. The torsion in $\mathbf{C}$ is $\mathbf{Q} f_{\lambda}+\mathbf{Q} g_{\lambda}$, and so our particular $\lambda$ gives a 
point of $Z \cap \mathbf{Q}^{4}$. It is even in $Z \cap \frac{1}{N} \mathbf{Z}^{4}$ if the torsion order divides $N$. Such points cannot be very numerous: in Wilkie's talks we saw that the cardinality

$$
\left|Z^{\text {trans }} \cap \frac{1}{N} \mathbf{Z}^{4} \cap K\right| \leq c(\epsilon, Z, K) N^{\epsilon}
$$

for a certain subset $Z^{\text {trans }}$ of $Z$ and any compact $K$ (maybe this could be eliminated using $o$-minimality) and any $\epsilon>0$. See also Pila [Pil] and Pila-Wilkie [PW]. It may be very difficult to write down $c(\epsilon, Z, K)$ in an effective way.

Here it is possible to show that $Z^{\text {trans }}=Z$; this is a concealed algebraic independence result as in Pila's talks, for which the hard (Hodge-theoretical) work was done by André [An].

We deal with $K$ using bounded height: more later. We get at most $c(\epsilon) N^{\epsilon}$ points $z_{\lambda}$. An easy argument with a faint flavour of zero-estimates of the type used in transcendence theory leads to at most $c(\epsilon) N^{\epsilon}$ values $\lambda$. But right now we don't know any upper bound for $N$.

In fact it is easy to see that these values $\lambda$ all lie in $\overline{\mathbf{Q}}$; further any given $\lambda$ yields $D=[\mathbf{Q}(\lambda): \mathbf{Q}]$ in all by conjugation $\lambda^{\sigma}$ (compare also Habegger's talks). We deduce

$$
D \leq c(\epsilon) N^{\epsilon}
$$

But there are also lower bounds for $D$. If we go back to the original problem of $\lambda, 1-\lambda$, now with say $\lambda$ of exact order $N_{1}$ then of course $D=\phi\left(N_{1}\right)$ for the Euler function, and this is classically known to be at least $c_{1}(\epsilon) N_{1}^{1-\epsilon}$ (now all constants are assumed positive). For our problem the analogue is that if $(2, \sqrt{4-2 \lambda})$ in $(3)$ has exact order $N_{1}$ then a famous Theorem of Serre [Se1] implies even

$$
D \geq C_{1} N_{1}^{2}
$$

(which is classical in the case of complex multiplication); but here the elliptic curve depends on $\lambda$ and therefore so does $C_{1}$. Furthermore it is not so easy to calculate this dependence. The work [MW2] (based on transcendence among other things) applies only if $N_{1}$ is prime, an assumption we cannot afford. It was extended to arbitrary $N_{1}$ by Zywina [Zy], but only for an elliptic curve defined over $\mathbf{Q}$, which we also cannot assume here. Very recently Lombardo [L] has extended the field of definition to $\overline{\mathbf{Q}}$; in a first version the dependence on $D$ was not quite good enough for application here, but he has since fixed this. There is also a dependence on the absolute height $h(\lambda)$ of $\lambda$. Fortunately a Theorem of Silverman [Si] 
implies that this height is bounded above by an absolute constant. Combining everything leads to $D \geq c N_{1}^{\delta}$ now with $c$ absolute. Here $\delta$ is less than $10^{-10}$.

For effectivity purposes it will probably be very convenient to have a bigger $\delta$. This arises from a more direct application of the transcendence methods, starting with an exponent smaller than 2 in (8). Then the very precise version [Davi2] due to David yields $D \geq c N_{1}^{1 / 2}$.

Similarly $D \geq c N_{2}^{1 / 2}$ for the exact order $N_{2}$ of $(3, \sqrt{18-6 \lambda})$ in (3). Taking $N$ now as the exact order, we have $N=\operatorname{lcm}\left(N_{1}, N_{2}\right) \leq N_{1} N_{2}$, and it follows that $D \geq c N^{1 / 4}$. Comparing this with (7), we see that it suffices to choose $\epsilon=\frac{1}{5}$ to get an absolute bound for $D=[\mathbf{Q}(\lambda): \mathbf{Q}]$. Combined with the absolute bound on $h(\lambda)$, this gives by a well-known result of Northcott (see below) the required finiteness.

As height bounds were not much mentioned in the other talks, we sketch here a proof that

$$
h(\lambda) \leq 6
$$

for the absolute height

$$
h(\lambda)=\frac{1}{D} \log \left(\left|a_{0}\right| \prod_{\sigma} \max \left\{1,\left|\lambda^{\sigma}\right|\right\}\right)
$$

where $a_{0} \lambda^{D}+\cdots=0$ is the minimal equation for $\lambda$ over $\mathbf{Z}$. All we use is $N_{1} P_{1}=0$ for $P_{1}=(2, \sqrt{4-2 \lambda})$, where the value of $N_{1} \geq 1$ is now irrelevant.

For any algebraic $\lambda$ and $P=(x, y)$ on $E_{\lambda}$ with algebraic $x, y$ we can reasonably define $h(P)=h(x)$ (but in Habegger's talks it was $h(x) / 2$ ), as $y$ is determined by $x$ and $\lambda$. For example $h\left(P_{1}\right)=\log 2$. The Néron-Tate height $\hat{h}(P)$ is defined for example by

$$
\hat{h}(P)=\lim _{k \rightarrow \infty} \frac{h\left(2^{k} P\right)}{4^{k}},
$$

and $|\hat{h}(P)-h(P)|$ is bounded above independently of $P$. Explicit bounds for Weierstrass elliptic curves are practically classical, but I calculated for Legendre

$$
|\hat{h}(P)-h(P)| \leq \frac{5}{3} h(\lambda)+c
$$

with explicit $c$ absolute.

Further $\hat{h}(P)=0$ if and only if $P$ is torsion. 
We look at $4 P_{1}$ on $E_{\lambda}$. With the help of classical duplication formulae we find

$$
4 P_{1}=\left(\frac{A(\lambda)}{B(\lambda)}, *\right)
$$

with $A, B$ in $\mathbf{Z}[t]$ of degrees 8 and 7 respectively. In fact

$$
\begin{gathered}
A=t^{8}-160 t^{7}+7104 t^{6}-57344 t^{5}+206336 t^{4}-401408 t^{3}+442368 t^{2}-262144 t+65536, \\
B_{4}=-288 t^{7}+3648 t^{6}-17408 t^{5}+38912 t^{4}-40960 t^{3}+16384 t^{2} .
\end{gathered}
$$

Now $h\left(\lambda^{8}\right)=8 h(\lambda)$ (not transparent from (9) by the way) and similarly one can show, after a bit of effort with resultants, that

$$
h\left(4 P_{1}\right) \geq 8 h(\lambda)-c^{\prime}
$$

with $c^{\prime}$ absolute.

On the other hand (10) gives

$$
h\left(4 P_{1}\right) \leq \hat{h}\left(4 P_{1}\right)+\frac{5}{3} h(\lambda)+c=\frac{5}{3} h(\lambda)+c
$$

SO

$$
h(\lambda) \leq \frac{c+c^{\prime}}{19 / 3}<6
$$

with some extra computation.

Here the Northcott result just mentioned becomes clear: if $h(\lambda)$ and $D$ are both bounded above, then so are $\left|a_{0}\right|$ and the $\left|\lambda^{\sigma}\right|$ in (9), and then so are the absolute values of the coefficients in the minimal polynomial $a_{0} \prod_{\sigma}\left(t-\lambda^{\sigma}\right)$.

This completes the sketch for (3) and $E_{\lambda}^{2}$. The general curve in $E_{\lambda}^{2}$ was treated in [MZ2], and more general products like $E_{\lambda} \times E_{-\lambda}$ in [MZ3]. For $A_{\lambda}$ as in [MZ4] and [MZ5] there are several extra technicalities. The results of André and Silverman apply also to the abelian situation. But despite the enormous advances by Serre in [Se2], still the extensions of [Se1] seem to be less clear-cut, even for powers of a fixed prime, let alone effective. But once more a transcendence approach succeeds, and we use David's result in [Davi1] pre-dating [Davi2]. In fact this result seems to require that the value $\lambda$ is such that $A_{\lambda}$ is simple. At first sight this looks like a problem. At second sight one suspects that such $\lambda$ are probably rare, possibly controlled by conjectures of André-Oort-Pink-Zilber type (see [Pin] and [Zi] for example). At third sight one realizes that such conjectures 
are not yet proved. But finally by going back into the proof in [Davi1] to winkle out the "obstruction subgroup" in the zero-estimate, one sees that some easy tricks from the geometry of numbers (as in [MW1] for example) suffice. One ends up with $D \geq c N^{\delta}$ with a ridiculously small exponent $\delta$ (depending on the dimension of $A_{\lambda}$ ). One could also use [MW3] to factorize the non-simple $A_{\lambda}$, but then the exponent would be even smaller. Probably recent work of Gaudron and Rémond [GR] would give more reasonable values.

Now for the applications of these results, denoted by (I) and (II) below.

(I) All know that Pell's equation

$$
x^{2}-d y^{2}=1, \quad y \neq 0
$$

is solvable over $\mathbf{Z}$ provided $d>0$ in $\mathbf{Z}$ is not a square. Moving to the polynomial ring $\mathbf{C}[t]$, by now a knee-jerk reaction, especially in view of "abcology", we consider

$$
X^{2}-D Y^{2}=1, \quad Y \neq 0
$$

with $D$ in $\mathbf{C}[t]$ not a square, surely easier. But in fact it is much more difficult to describe the set of $D$ for which there is solvability over $\mathbf{C}[t]$. One can easily see that the degree $m$ of $D$ must be even. Now we proceed systematically.

$\underline{m=2}$ : there is always solvability. Thus for $D=a t^{2}+b t+c$ we can take

$$
X=\frac{2 a t+b}{\sqrt{b^{2}-4 a c}}, \quad Y=\frac{2 \sqrt{a}}{\sqrt{b^{2}-4 a c}} .
$$

$\underline{m=4}$ : there is not always solvability. For example not for $D=t^{4}+t+1$. And in the family $D=t^{4}+t+\lambda$ we have solvability exactly when $\lambda$ lies in a certain countable subset of $\mathbf{C}$. In fact precisely when the point $(0,1)$ is torsion on $y^{2}=x^{3}-4 \lambda x+1$. This is essentially classical (Abel $[\mathrm{Ab}]$, Chebychev [C1],[C2]). In fact the set is infinite (which is not classical - for several proofs see [Za] pages 92,93 for example), as one might guess from its element (with its six conjugates)

$$
\lambda=\frac{\sqrt[3]{2 \sqrt{2}-2}}{2}
$$

where

$$
X=\frac{\left(4-32 \lambda^{3}\right) t^{5}-\left(4 \lambda-16 \lambda^{4}\right) t^{4}+4 \lambda^{2} t^{3}+\left(3-28 \lambda^{3}\right) t^{2}-8 \lambda^{4} t+8 \lambda^{5}}{32 \lambda^{8}},
$$




$$
Y=\frac{\left(4-32 \lambda^{3}\right) t^{3}-\left(4 \lambda-16 \lambda^{4}\right) t^{2}+4 \lambda^{2} t+\left(1-12 \lambda^{3}\right)}{32 \lambda^{8}} .
$$

$\underline{m=6}$ : there is rarely solvability. For example there are only finitely many $\lambda$ in $\mathbf{C}$ for which solvability holds for $D=t^{6}+t+\lambda$. This is proved in [MZ4] using the general result on $A_{\lambda}$ described above. In fact here $A_{\lambda}$ is the Jacobian of the hyperelliptic curve $s^{2}=t^{6}+t+\lambda$ of genus 2 , or better a complete non-singular model

$$
s^{2}=t_{3}^{2}+t_{0} t_{1}+\lambda t_{0}^{2}, \quad t_{0} t_{2}=t_{1}^{2}, \quad t_{0} t_{3}=t_{1} t_{2} .
$$

The curve inside $A_{\lambda}$ is the locus of the divisor $\Delta_{\lambda}=\infty_{\lambda}^{+}-\infty_{\lambda}^{-}$as $\lambda$ varies, where $\infty_{\lambda}^{ \pm}$are the two places at infinity. When (11) holds we write it as $f_{\lambda}^{+} f_{\lambda}^{-}=1$ with the functions $f_{\lambda}^{ \pm}=X \pm s Y$ to see that their divisors are multiples of $\Delta_{\lambda}$ thus giving a torsion point.

There are actually some $\lambda$; for example $\lambda=0$ with

$$
X=2 t^{5}+1, \quad Y=2 t^{2}
$$

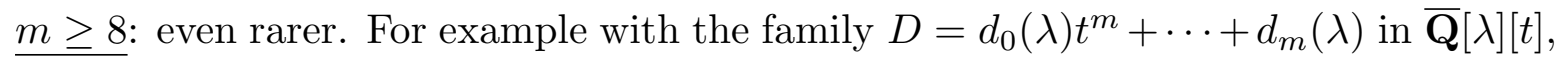
say for safety identically squarefree, there is solvability for infinitely many $\lambda$ in $\overline{\mathbf{Q}}$ only if the analogous Jacobian, now an abelian variety of dimension $\frac{m-2}{2} \geq 3$, contains an elliptic curve. This also follows from the $A_{\lambda}$ result in [MZ5].

Incidentally, if we want to go beyond squarefree, then we can use the result of [BMPZ] on multiplicative extensions (4). Thus for $D=t^{2}\left(t^{4}+t+\lambda\right)$ we get at most a finite set, despite the infinite set for $t^{4}+t+\lambda$. And also for $D=t^{3}\left(t^{3}+t+\lambda\right)$ using the additive extensions (6).

(II) This concerns the old problem of "integrating in elementary terms" (see for example the article $[\mathrm{R}]$ by Risch). By the way, the integration may be elementary but it need not be easy (just as for some proofs), as a wonderful example

$$
\int \frac{\sqrt{1+t^{4}}}{1-t^{4}} d t=-\frac{1}{4} \sqrt{2} \log \left(\frac{\sqrt{2} t-\sqrt{1+t^{4}}}{1-t^{2}}\right)-\frac{i}{4} \sqrt{2} \log \left(\frac{i \sqrt{2} t+\sqrt{1+t^{4}}}{1+t^{2}}\right)
$$

due to Euler shows. Not only can my Maple (version 9) not do the integration on the left-hand side, but it cannot even check the result by differentiating the right-hand side. Actually Euler's version was

$$
\frac{1}{4} \sqrt{2} \log \left(\frac{\sqrt{2} t+\sqrt{1+t^{4}}}{1-t^{2}}\right)+\frac{1}{4} \sqrt{2} \arcsin \left(\frac{\sqrt{2} t}{1+t^{2}}\right)
$$


thus staying over $\mathbf{R}$.

We give some more examples in the above hyperelliptic context.

$\underline{m=2}$ : now $\int \frac{d t}{\sqrt{D}}$ is always integrable - see any engineer's handbook of indefinite integrals.

$\underline{m=4}:$ now $\int \frac{d t}{\sqrt{t^{4}+t+\lambda}}$ is integrable if and only if $256 \lambda^{3}=27$; then we can reduce it to

$$
\int \frac{d t}{(t+4 \lambda / 3) \sqrt{t^{2}+\mu t+\nu}}
$$

and run to the handbook.

$\underline{m=6}$ : now the same methods show that $\int \frac{d t}{\sqrt{t^{6}+t+\lambda}}$ is integrable only if $46656 \lambda^{5}=$ 3125 , and with a bit more effort never.

However up to now all that is in fact relatively easy, and not at the same level as Pell. But

$$
\int \frac{d t}{t \sqrt{t^{4}+t+\lambda}}
$$

is integrable if and only if $\lambda$ lies in a certain finite set. Oddly enough the proof does not use multiplicative extensions as for $t^{2}\left(t^{4}+t+\lambda\right)$ above but rather Schmidt's result [Sc] on additive extensions (6). Incidentally, he has made such results effective, using among other things a version [Ma] of the original result of Bombieri-Pila [BP] obtained as in Wilkie's talks with the Siegel Lemma). For example he shows that there are at most $e^{e^{e^{e^{5}}}}$ complex values of $\lambda$ for which

$$
\int \frac{d t}{(t-2) \sqrt{t(t-1)(t-\lambda)}}
$$

is integrable. This is related to (3): thus integrability implies that $(2, \sqrt{4-2 \lambda})$ is torsion on $E_{\lambda}$. But the converse fails, so we cannot deduce infinitely many $\lambda$. In fact we get a torsion point even on a suitable $G_{\lambda}$ as in (6), so we may conclude finiteness.

And also by [MZ5]

$$
\int \frac{d t}{t \sqrt{t^{6}+t+\lambda}}
$$

is integrable at most on a finite set; but no-one knows how to make this effective. Here we use $A_{\lambda}$ as above, but now with the locus of $\Gamma_{\lambda}=P_{\lambda}^{+}-P_{\lambda}^{-}$, where $P_{\lambda}^{ \pm}=(0, \pm \sqrt{\lambda})$; this time the torsion property arises from a classical criterion of Liouville, which implies that the integral, if elementary, must involve a single $\log g_{\lambda}$ (as opposed to (12) with a pair). 
Now differentiation (without Maple) gives $d t /(t s)=c d g_{\lambda} / g_{\lambda}$ for $c$ in $\mathbf{C}$, from which we see that the divisor of $g_{\lambda}$ is a multiple of $\Gamma_{\lambda}$.

These examples support an assertion of James Davenport [Dave] from 1981.

I thank Gareth Jones for comments on an earlier version.

\section{References}

[Ab] N.H. Abel, Über die Integration der Differential-Formel $\rho d x / \sqrt{R}$, wenn $R$ und $\rho$ ganze Funktionen sind, J. für Math. (Crelle) 1 (1826), 185-221.

[An] Y. André, Mumford-Tate groups of mixed Hodge structures and the theorem of the fixed part, Compositio Math. 82 (1992), 1-24.

[Be] D. Bertrand, Special points and Poincaré bi-extensions; with an Appendix by Bas Edixhoven, ArXiv 1104.5178v1.

[BMPZ] D. Bertrand, D. Masser, A. Pillay, and U. Zannier, Relative Manin-Mumford for semi-abelian surfaces, submitted for publication.

[BP] E. Bombieri and J. Pila, The number of integral points on arcs and ovals, Duke Math. J. 59 (1989), 337-357.

[C1] P. Chebychev, Sur l'intégration des différentielles qui contiennent une racine carée d'un polynome du troisième ou du quatrième degré, J. Math. Pures Appl. 2 (1857), 168-192.

[C2] P. Chebychev, Sur l'intégration de la différentielle $\frac{x+A}{\sqrt{x^{4}+\alpha x^{3}+\beta x^{2}+\gamma x+\delta}}$, J. Math. Pures Appl. 9 (1864), 225-246.

[Dave] J.H. Davenport, On the integration of algebraic functions, Lecture Notes in Computer Science 102, Springer-Verlag, Berlin Heidelberg New York 1981.

[Davi1] S. David, Fonctions theta et points de torsion des variétés abéliennes, Compositio Math. 78 (1991), 121-160.

[Davi2] S. David, Points de petite hauteur sur les courbes elliptiques, J. Number Theory 64 (1997), 104-129.

[GR] É. Gaudron and G. Rémond, Théorème des périodes et degrés minimaux d'isogénies, to appear in Commentarii Math. Helv. (39 pages). 
[H] M. Hindry, Autour d'une conjecture de Serge Lang, Inventiones Math. 94 (1988), 575-604.

[L] D. Lombardo, Bounds for Serre's open image theorem for elliptic curves over number fields, preprint 2014 (36 pages).

[Ma] D. Masser, Rational values of the Riemann zeta function, J. Number Theory 131 (2011), 2037-2046.

[MW1] D. Masser and G. Wüstholz, Periods and minimal abelian subvarieties, Ann. of Math. 137 (1993), 407-458.

[MW2] D. Masser and G. Wüstholz, Galois properties of division fields of elliptic curves, Bull. London Math. Soc. 25 (1993), 247-254.

[MW3] D. Masser and G. Wüstholz, Factorization estimates for abelian varieties, Publ. Math. IHES 81 (1995), 5-24.

[MZ1] D. Masser and U. Zannier, Torsion anomalous points and families of elliptic curves, Amer. J. Math. 132 (2010), 1677-1691.

[MZ2] D. Masser and U. Zannier, Torsion points on families of squares of elliptic curves, Math. Annalen 352 (2012), 453-484.

[MZ3] D. Masser and U. Zannier, Torsion points on families of products of elliptic curves, to appear in Advances in Math.

[MZ4] D. Masser and U. Zannier, Torsion points on families of abelian surfaces and Pell's equation over polynomial rings (with Appendix by V. Flynn), submitted for publication.

[MZ5] D. Masser and U. Zannier, Torsion points on families of abelian varieties, Pell's equation, and integration in elementary terms, in preparation.

[Pil] J. Pila, Integer points on the dilation of a subanalytic surface, Quart. J. Math. 55 (2004), 207-223.

[PW] J. Pila and A. Wilkie, The rational points of a definable set, Duke Math. J. 133 (2006), 591-616.

[PZ] J. Pila and U. Zannier, Rational points in periodic analytic sets and the ManinMumford conjecture, Rendiconti Lincei Mat. Appl. 19 (2008), 149-162.

[Pin] R. Pink, A common generalization of the conjectures of André-Oort, Manin-Mumford, and Mordell-Lang, manuscript dated 17th April 2005 (13 pages). 
[R] R.H. Risch, The problem of integration in finite terms, Trans. Amer. Math. Soc. 139 (1969), 167-189.

[Sc], H. Schmidt, Ph.D. thesis (Basle), in preparation.

[Se1] J-P. Serre, Propriétés galoisiennes des points d'ordre fini des courbes elliptiques, Inventiones Math. 15 (1972), 259-331.

[Se2] J-P. Serre, Résumé des cours au Collège de France de 1985-1986, Collected papers IV 1985-1998, Springer-Verlag, Berlin 2000.

[Si] J.H. Silverman, Heights and the specialization map for families of abelian varieties, J. reine angew. Math. 342 (1983), 197-211.

[Za] U. Zannier, Some problems of unlikely intersections in arithmetic and geometry, Annals of Math. Studies 181, Princeton 2012.

[Zi] B. Zilber, Exponential sums equations and the Schanuel conjecture, J. London Math. Soc. 65 (2002), 27-44.

[Zy] D. Zywina, Bounds for Serre's open image theorem, ArXiv e-prints, 1102.4656, February 2011 (16 pages).

D. Masser: Mathematisches Institut, Universität Basel, Rheinsprung 21, 4051 Basel, Switzerland (David.Masser@unibas.ch).

Submitted 27th April 2014.

Revised 11th June 2014. 


\section{LATEST PREPRINTS}

No. Author: Title

2014-01 Helmut Harbrecht, Michael Peters, Markus Siebenmorgen

Efficient Approximation of Random Fields for Numerical Applications

2014-02 Ali Hyder, Luca Martinazzi

Conformal Metrics on $R^{2 m}$ with Constant Q-Curvature, Prescribed Volume and Asymptotic Behavior

2014-03 Jürgen Dölz, Helmut Harbrecht, and Michael Peters

H-Matrix Accelerated Second Moment Analysis for Potentials with Rough Correlation

2014-04 Tianling Jin, Ali Maalaoui, Luca Martinazzi, Jingang Xiong Existence and Asymptotics for Solutions of a Non-Local Q-Curvature Equation in Dimension Three

2014-05 Marcus J. Grote, Michaela Mehlin, Teodora Mitkova

Runge-Kutta Based Explicit Local Time-Stepping Methods for Wave Propagation

2014-06 Hanspeter Kraft, Andriy Regeta

Automorphisms of the Lie Algebra of Vector Fields on Affine n-Space

2014-07 Jérémy Blanc, Alberto Calabri

On Degenerations of Plane Cremona Transformations

2014-08 Helmut Harbrecht, Michael Peters, Markus Siebenmorgen

Numerical Solution of Elliptic Diffusion Problems on Random Domains

2014-09 H. Harbrecht, W.L. Wendland, and N. Zorii

Rapid Solution of Minimal Riesz Energy Problems

2014-10 Julien Diaz, Marcus J. Grote

Multi-Level Explicit Local Time-Stepping Methods for Second-Order Wave Equations

2014-11 J. Dölz, H. Harbrecht, Ch. Schwab

Covariance Regularity and H-Matrix Approximation for Rough Random Fields

2014-12 Giovanni Alberti, Gianluca Crippa, Anna L. Mazzucato

Exponential Self-Similar Mixing and Loss of Regularity for Continuity

Equations

Preprints are available under http://math.unibas.ch/research/publications/ 


\section{LATEST PREPRINTS}

No. Author: Title

2014-13 M. Dambrine, H. Harbrecht, B. Puig

Computing Quantities of Interest for Random Domains with Second Order Shape Sensitivity Analysis

2014-14 Monica Bugeanu, Helmut Harbrecht

A Second Order Convergent Trial Method for free Boundary Problems in Three Dimensions

2014-15 David Masser

Relative Manin-Mumford for Abelian Varieties 